\title{
Quantifying the value of redundant measurements at GCOS Reference Upper-Air Network sites
}

\author{
F. Madonna ${ }^{1}$, M. Rosoldi ${ }^{1}$, J. Güldner ${ }^{2}$, A. Haefele ${ }^{3}$, R. Kivi ${ }^{4}$, M. P. Cadeddu ${ }^{5}$, D. Sisterson ${ }^{5}$, and G. Pappalardo ${ }^{1}$ \\ ${ }^{1}$ Consiglio Nazionale delle Ricerche, Istituto di Metodologie per l'Analisi Ambientale (CNR-IMAA), C. da S. Loja, \\ Tito Scalo, Potenza, 85050, Italy \\ ${ }^{2}$ Deutscher Wetterdienst, Meteorologisches Observatorium Lindenberg Richard Assmann Observatorium, \\ Am Observatorium 12, 15848 Tauche/Lindenberg, Germany \\ ${ }^{3}$ Federal Office of Meteorology and Climatology MeteoSwiss, Chemin de l'Aérologie, 1530 Payerne, Switzerland \\ ${ }^{4}$ Finnish Meteorological Institute, Arctic Research, Tähteläntie 62, 99600 Sodankylä, Finland \\ ${ }^{5}$ Argonne National Laboratory, 9700 South Cass Avenue, Argonne, IL 60439-4801, USA
}

Correspondence to: F. Madonna (fabio.madonna@imaa.cnr.it)

Received: 28 April 2014 - Published in Atmos. Meas. Tech. Discuss.: 23 June 2014

Revised: 11 September 2014 - Accepted: 24 September 2014 - Published: 19 November 2014

\begin{abstract}
The potential for measurement redundancy to reduce uncertainty in atmospheric variables has not been investigated comprehensively for climate observations. We evaluated the usefulness of entropy and mutual correlation concepts, as defined in information theory, for quantifying random uncertainty and redundancy in time series of the integrated water vapour (IWV) and water vapour mixing ratio profiles provided by five highly instrumented GRUAN (GCOS, Global Climate Observing System, Reference Upper-Air Network) stations in 2010-2012. Results show that the random uncertainties on the IWV measured with radiosondes, global positioning system, microwave and infrared radiometers, and Raman lidar measurements differed by less than $8 \%$. Comparisons of time series of IWV content from ground-based remote sensing instruments with in situ soundings showed that microwave radiometers have the highest redundancy with the IWV time series measured by radiosondes and therefore the highest potential to reduce the random uncertainty of the radiosondes time series. Moreover, the random uncertainty of a time series from one instrument can be reduced by $\sim 60 \%$ by constraining the measurements with those from another instrument. The best reduction of random uncertainty is achieved by conditioning Raman lidar measurements with microwave radiometer measurements. Specific instruments are recommended for atmospheric water vapour measurements at GRUAN sites. This
\end{abstract}

approach can be applied to the study of redundant measurements for other climate variables.

\section{Introduction}

The use of redundant measurements is considered the best approach to reduce the uncertainty of an atmospheric variable. For this reason, several atmospheric observatories have extended their observing capabilities and have acquired multiple instruments that measure the same atmospheric variables with different measurement techniques and retrieval algorithms.

Redundancy can be defined as the duplication or the multiplication of the estimation of an atmospheric variable with the aim of increasing reliability in the study of the same variable over the time. Without doubt, redundant measurements provide added value towards the full exploitation of the synergy among different measurements techniques: the main advantages are related to

- filling gaps and improving measurement continuity over time and vertical range;

- increasing the sampling rate by merging measurements from different instruments; 
- addressing instrument noise and identifying possible biases or retrieval problems by comparing different techniques and instruments.

Comprehensive studies to quantify the effective value of redundant measurements and their ability to reduce uncertainty of essential climate variables (ECVs), as retrieved by multiple ground-based techniques and in situ active and passive remote sensing, are missing. To this end, GRUAN (GCOS, Global Climate Observing System, Reference Upper-Air Network) aims at providing long-term, highly accurate measurements of atmospheric profiles, complemented by surface-based state-of-the-art instrumentation, for full characterization of ECVs and their changes in the complete atmospheric column (Seidel et al., 2009; Thorne et al., 2013). GRUAN, which is now being implemented, is aimed at supporting a network of 30-40 high-quality, long-term upperair observing stations, building on existing observational networks.

Cross-checking of redundant measurements for consistency is an essential part of the GRUAN quality assurance procedures. A fully equipped GRUAN site should make at least three redundant measurements of all GCOS ECVs (Seidel et al., 2008). As a consequence, the GRUAN community has fostered GATNDOR (GRUAN Analysis Team for Network Design and Operations Research), a scientific team charged with addressing key scientific questions of major interest to GRUAN and identifying reliable metrics for quantifying the value of redundant measurements.

The present study used observations of the vertical-profile of water vapour mixing ratio and the integrated water vapour (IWV) content from a few GRUAN sites equipped with radiosondes, global positioning system (GPS), lidars, radiometers, spectrometers, and radars. Studies of redundant measurements should be based on the preliminary identification of a reliable metric. Linear correlation (Pearson's or Spearman's) has typically been used to study redundant measurements and their reliability. More recently, Fassò et al. (2014) presented a new approach for an advanced statistical modelling based on functional data analysis of the relationships among collocation uncertainty and a set of environmental factors (e.g. wind speed and wind direction). The approach, which can decompose the total collocation uncertainty, could be adapted to evaluate the measurement redundancy. In this paper, we present the results of the GATNDOR study of redundant measurements at GRUAN sites. The present study identifies mutual correlation (MC), which is related to the concept of entropy, as a suitable metric for quantifying the value of measurement redundancy. In information theory, entropy is a measure of the probabilistic uncertainty associated with a random variable. The approach presented here represents a fast, efficient way to quantify the value of redundant measurements and to correlate the value with factors such as number of instruments, as reported in this work, type of measurement techniques, and retrieval algorithms.
The aims of the paper are

- to show the potential of entropy and MC as metrics for quantifying uncertainty (in a probabilistic sense) and the value of redundancy in climate time series;

- to study, according to GRUAN standards, the uncertainty and the value of redundancy of in situ and groundbased remote sensing techniques for estimating ECVS;

- to provide the GRUAN community and others interested in the observation of atmospheric thermodynamics with recommendations for the establishment of an observation protocol to reduce the uncertainty of a measurement time series through measurement redundancy;

- to aid site scientists, managers, and funders in making informed decisions on new instrument procurements to maximize the scientific return on the capital expenditure.

Section 2 outlines information theory concepts used for the study of redundancy and presents the data sets considered in this work. The data sets were provided by five candidate GRUAN sites: the Atmospheric Radiation Measurement (ARM) Program Southern Great Plains in Oklahoma, USA (Miller et al., 2003); CIAO (Consiglio Nazionale delle Ricerche, Istituto di Metodologie per l'Analisi Ambientale (CNR-IMAA) Atmospheric Observatory) in Potenza, Italy (Madonna et al., 2011); Lindenberg in Germany (Adam et al., 2005); Payerne in Switzerland (Calpini et al., 2011); and Sodankylä in Finland (Hirsikko et al., 2014). Section 3 provides results and preliminary remarks on the value of redundant measurements in reducing uncertainty and introduces a possible criterion for addressing redundancy in the frame of GRUAN. Section 4 summarizes the conclusions.

\section{Methodology}

\subsection{Comparison methods}

Comparisons among time series of in situ and ground-based remote sensing measurements have been performed mostly by using the concept of variance and root-mean-square difference, less frequently in terms of "information" content (e.g. Majda and Gershgorin, 2010). In information theory, as in thermodynamics, entropy is a measure of the number of specific ways a system can be arranged. Entropy is often considered a measure of disorder or uncertainty in the outcome or the prediction of an event. Commonly used in time series analysis is the Shannon-Wiener entropy measure (Cover and Thomas, 1991). Given $x$ events in the population $X$ occurring with probabilities $p(x)$, the Shannon entropy is defined as

$H(x)=-\sum_{x \in X} p(x) \log (p(x))$. 
Therefore, $H$ is a measure of probabilistic uncertainty or dispersion of the probabilities of events. The entropy is calculated from a histogram of probabilities; it has a maximum value if all measurements have equal probability of occurrence and a minimum value of 0 if the probability of one measurement is 1 and the probability of all the others is 0 . The $H$ is not equivalent to variance $(\sigma)$, though for particular classes of distributions (e.g. Gaussian), $H$ is simply some function of $\sigma$, and they can be considered almost equivalent. Entropy generalizes the concept of measurement uncertainty for calculations of MC. Normalized $H$ is used here to quantify the uncertainty of a time series, and $H$ is normalized by dividing $H$ by the logarithm of the number of states (i.e. the number of possible entries in the related histogram).

In information theory, MC is a measure of the statistical dependence of two random variables or, equivalently, the amount of information that one variable contains about the other (Cover and Thomas, 1991). The MC value can be considered a qualitative indication of how well one measurement explains the other. This means that MC quantifies the reduction of uncertainty in a variable $Y$ after one observes another variable $X$. The advantage in using MC with respect to Pearson's or Spearman's correlation coefficient $(\rho)$ is that MC is a more general measure than $\rho$, because it does not assume linear or even monotonic correlation. Entropy and mutual information are both rather insensitive to outliers, but even a single outlier can arbitrarily impact both the variance and correlation between two distributions, obscuring the similarity of two closely related variables.

The MC of two discrete random variables $X$ and $Y$ can be defined as (Cover and Thomas, 1991)

$\operatorname{MC}(X, Y)=\sum_{y \in Y} \sum_{x \in X} p(x, y) \log \left(\frac{p(x, y)}{p(x) p(y)}\right)$,

where $p(x, y)$ is the joint probability distribution function of $X$ and $Y$, and $p(x)$ and $p(y)$ are the marginal probability distribution functions of $X$ and $Y$, respectively. For continuous random variables, the summation is implemented with a definite double integral. The redundancy concept is a generalization of mutual information to $N$ variables $\left(X_{1}, X_{2}, \ldots, X_{N}\right)$. Given as marginal entropies $H(X)$ and $H(Y)$, MC can be also defined as

$\operatorname{MC}(X, Y)=H(X)+H(Y)-H(X, Y)$.

The joint entropy $H(X, Y)$ is the total amount of information for two time series and is calculated by using the joint histogram of the two series. If the two measurements are totally unrelated, then the joint entropy will be the sum of the entropies of the individual measurements. In general, $H(X, Y) \leq H(X)+H(Y)$. The entropy gained from a member of a mixture of distributions is the difference between the entropy of the average distribution and the average of the entropies of the individual distributions. $H(X, Y)$ can be calculated by using the joint histogram of $X$ and $Y$.
The MC can be also linearized; differences between nonlinear and linear redundancy provide a qualitative test for the non-linearity of the investigated problem. The linear MC is defined as (Cover and Thomas, 1991)

$\mathrm{LMC}=\frac{1}{2} \sum_{i=1}^{m} C_{i i}-\frac{1}{2} \sum_{i=1}^{m} \lambda_{i}^{C}$,

where the $C_{i i}$ values are the diagonal elements of the covariance matrix $\mathbf{C}$ of the $m$ time series investigated, and the $\lambda$ values are the eigenvalues of $\mathbf{C}$. A comparison between linear and non-linear MC is in Sect. 3.4.

Many applications require a metric - a distance measure not only between points but also between data clusters (or time series of data). Different distances are defined in the literature (Arkhangel'skii and Pontryagin, 1990). Here, $D$ is defined as

$D(X, Y)=1-\operatorname{MC}(X, Y) / \max (H(X), H(Y))$,

where $D$ is a metric that satisfies the triangle inequality (i.e. given $X, Y, Z$, the sum of $D$ of any two of the considered variables must be greater than or equal to the value of $D$ for the remaining variable). Calculation of $\mathrm{MC}$ is an effective way to compare clustering and study relationships between time series (Correa and Lindstrom, 2012).

Finally, the conditional entropy is defined as $H(X \mid Y)=$ $H(Y)-\operatorname{MC}(X, Y)$. This definition can be generalized for two or more conditioning variables through the chain rule for joint entropy (Cover and Thomas, 1991).

\subsection{Data sets and instruments}

The data sets considered in this study include radiosonde, Raman lidar, infrared and microwave radiometry (MWR) observations from the GRUAN sites (Lindenberg, Payerne, Potenza, Sodankyla and ARM Southern Great Plains). The data sets are collected by each station according to their quality assurance criteria. More information about the selected sites can be found at www.gruan.org. This study focused on the investigation of atmospheric water vapour measurements, both profiling and columnar, from these sites for 2010-2012. The instruments considered at the five selected sites are identified in Table 1. It is important to note that not all the considered station are routinely providing the uncertainties related to each instrument. However (to help the reader in the interpretation of the results), typical uncertainties affecting the considered measurements are mentioned: radiosonde water vapour mixing ratio profiles have a relative uncertainty typically lower than $6 \%$ from the surface until $15 \mathrm{~km}$ a.g.1., though the uncertainty might change depending on the measurements conditions (more details in Dirksen et al., 2014); Raman lidar relative random uncertainty increases with height and, for the profiles used in this study, it is less than $25 \%$ at $7-8 \mathrm{~km}$ a.g.l. plus a calibration error typically within $5-10 \%$ affecting the entire profile; the uncertainty 
on the integrated water vapour content achievable with microwave radiometers and profilers is strongly dependent on the retrieval types, but it is typically within about $\pm 0.07 \mathrm{~cm}$; the GPS uncertainty on the integrated water vapour content is typically within about $\pm 0.15 \mathrm{~cm}$ (first results from GRUAN comparisons with $\mathrm{CFH}$ ). GRUAN is establishing a database of ECV measurements from the different techniques and instruments, including full characterization of the uncertainty budget (random and bias contributions). The added value of GRUAN products is related to the implementation of data processing including several corrections for spurious effects on the radiosonde measurements and therefore on the fidelity of the long-term records of radiosondes used for climate applications (Immler et al., 2010; Immler and Sommer, 2010). At present, only quality-assured measurements obtained by RS92-SGP sondes are flowing into the GRUAN data archive. Unfortunately, the approach presented in this paper cannot be used to show the advantages of using GRUAN sonde products, mainly because the bias component of the total uncertainty budget cannot be quantified through the entropy analysis presented here.

Water vapour measurements from sensors not considered in this study are also available for the considered sites (as noted in Table 1); they are a subject for future study. The current water vapour measurements were selected according to data availability for each site. A similar investigation could be performed for other ECVs. For coherency, we used sonde data processed at each site rather than GRUAN products, which are still not available at all sites and for all radiosonde types. Moreover, retrieval algorithms for passive instruments usually take advantage of historical radiosonde data sets as a statistical constraint.

Simultaneous data from all available instruments were selected according to the conditions of clear sky (per lidar measurements or radiosonde humidity), nighttime, and, if lidar data are available, a relative error of lidar water vapour mixing ratio at $7 \mathrm{~km}$ a.g.l. $<25 \%$. This error is considered a good compromise having an adequate lidar signal-to-noise ratio and also covering the part of the troposphere where most of the water vapour can be observed. Raman lidar measurements are integrated over $10 \mathrm{~min}$ around the sonde synoptic launch time to keep a good signal-to-noise ratio in the investigated region, and MWR and microwave profiler measurements are provided every $10 \mathrm{~min}$. GPS data are provided only every $15 \mathrm{~min}$, because of constraints on data processing at the considered sites, and the closest measurements to the sonde launch time (within $10 \mathrm{~min}$ ) are compared. The use of MWR to calibrate the ARM Raman lidar measurements affects the independence of the IWV comparison for lidar at the SGP; in contrast, at Payerne and Potenza the Raman lidar is calibrated by using radiosonde humidity profiles in the lower troposphere (Madonna et al., 2011; Brocard et al., 2013).

Data from different sites are currently processed with different algorithms; this could affect the comparison. However, the study of entropy is also a good check for the effect of re- trieval inconsistencies. A linear regression on the entire time series (3 years) of IWV data and vertical profiles of water vapour mixing ratio at the altitude levels removed natural or artificial trends (e.g. calibration drifts). This was done to suppress the bias component of the time series uncertainty and to ensure that the reported entropies are related only to the random uncertainty.

\subsection{Optimal binning choice and minimally sufficient data}

The two crucial issues that need to be considered for entropy calculation using the histogram of a variable are the minimal quantity of data required to reduce inaccuracies in the calculation and the choice of the optimal binning to represent the actual probability density functions (PDFs) of the variable.

To make our histogram representative of the real underlying PDF of the variable and to calculate the related entropy, a minimal number of data points is needed. The data sets considered here include $>140$ cases per station (Lindenberg $=296$, Payerne $=174$, Southern Great Plains $=144)$. For Potenza and Sodankyla, more restricted data sets (40 and 22 cases, respectively) were used, because of the unique sampling strategy at Potenza (one radiosonde launch per week, only in clear sky) and the limited number of cryogenic frost point hygrometer $(\mathrm{CFH})$ launches made available by Sodankyla for this study. Knuth (2013) reported that at least 100 cases should be considered to avoid underestimation of entropy, though the number might depend on the underlying distribution. Nevertheless, values of the entropy calculated for Potenza and Sodankyla are quite similar to those reported for other sites. This is encouraging, though a margin of inaccuracy affecting the values can be quantified only if larger data sets become available for the specific instruments at both stations.

To determine the optimal binning, several statistical methods have been proposed (Knuth, 2013). In Fig. 1, entropy is shown as a function of the number of bins used to build the histogram for the Payerne radiosonde data sets. The value of entropy increases up to 0.81 for a histogram with 100 bins. Between 25 and 100 bins, entropy tends to assume asymptotic behaviour. In this work, in view of the behaviour shown in Fig. 1 and the number of data points available, 50 bins per histogram are used.

\section{Results and discussion}

In this section, normalized entropy, $\mathrm{MC}$, and conditional entropy are calculated for the data sets (and instruments) identified in Table 1. Both quantities were calculated to quantify uncertainty and redundancy in the IWV time series, as well as in the times series of the vertical profile of water vapour mixing ratio. In this investigation of time series of atmospheric water vapour measurements, entropy includes 
Table 1. Instruments available (and model when applicable) at the GRUAN sites generating data sets considered in this study of uncertainty and redundancy. Symbol ! indicates that the instrument is available at the site, but the data were not used in the study. Abbreviations: $\mathrm{CFH}$, cryogenic frost-point hygrometer; MWR, microwave radiometer; MWP, microwave profiler; GPS, global positioning system; FTIR, Fourier transform infrared radiometer; AERI, atmospheric emitted radiance interferometer.

\begin{tabular}{llcclccc}
\hline GRUAN site/instrument & Sonde & CFH & Lidar & MWR & MWP & GPS & FTIR \\
\hline Lindenberg & RS-92 $(4 \times$ day $)$ & $!$ & X & Radiometrics & Radiometrics & GFZ \\
Payerne & SRS 400 $(2 \times$ day $)$ & & X & HATPRO & & GFZ \\
Potenza & RS-92 $(1 \times$ week $)$ & & X & & Radiometrics & ! \\
Sodankyla & RS-92, I-Met1 RSB & X & $!$ & & & ! & Bruker \\
Southern Great Plains & RS-92 $(4 \times$ day $)$ & & X & Radiometrics & & Suominet & AERI \\
\hline
\end{tabular}

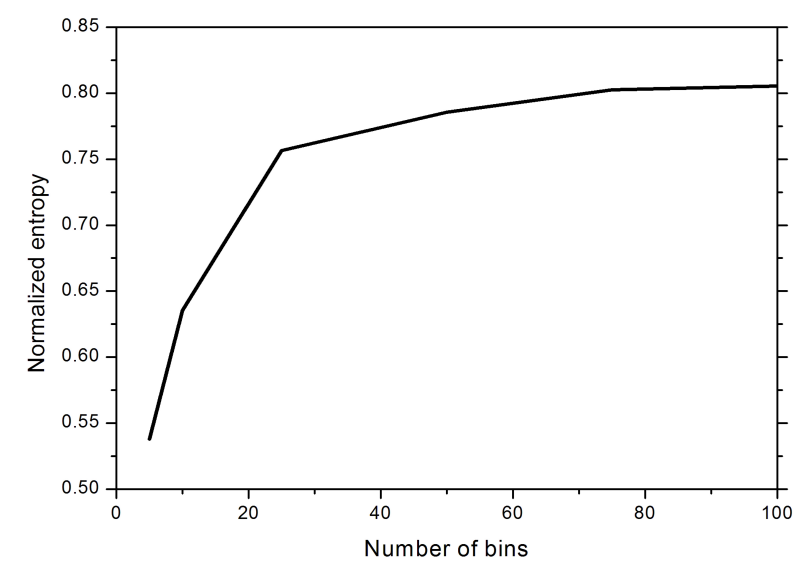

Figure 1. Entropy as a function of the number of bins used to build the histogram for the Payerne radiosonde.

all contributions affecting the uncertainty of a measurements time series - sampling uncertainty, uncertainty due to the time and vertical average, atmospheric variability, and all other relevant environmental factors (Kitchen, 1989; Fassò et al., 2014), such as solar radiation affecting daytime in situ soundings.

Figure 2 (left) is an example of a series of samples of the IWV for the Lindenberg instruments (Table 1), while Fig. 2 (right) shows the corresponding histograms of the time series. After linear detrending of the time series described above, the histograms were used to calculate entropy and MC. The shape of the histograms in Fig. 2 clarifies both how outliers can occur by chance in any distribution, often indicating either measurement errors or a heavy-tailed distribution in the population, and also the absence of any guarantee that the distribution will be a normal one. The discrepancies between the time series reported in Fig. 2 (left) translate into a sort of bi-modal distribution characterized by a high kurtosis (Fig. 2, right). Thus, caution is needed in assuming a normal distribution; statistics, like entropy, that are robust to outliers and independent on the underlying distribution are more reliable for characterizing the uncertainty of a time series.
To show the reader the advantages of using entropy and MC instead of using standard deviation $(\sigma)$ and $\rho$, we show in Fig. 3 a Taylor diagram (left panel) obtained from the GPS IWV time series collected at Lindenberg, and the same time series but adding to the IWV probability density function 5 , $10,20,30$, and 40 outliers respectively. The correlation has been calculated with respect to an underlying Gaussian distribution fitted to the data. The value of the $\sigma$ in the diagram obtained from the original time series is reported as the "observed" curve. Taylor diagrams provide a concise statistical summary of the similarity between two patterns, quantified in terms of their correlation, their centred root-mean-square difference and the amplitude of their variations (represented by their $\sigma$ s). These diagrams are especially useful in evaluating multiple aspects of complex models or in gauging the relative skill of many different models or measurement techniques.

Following previous studies, the Taylor diagram described above is compared in Fig. 3 with a modified Taylor diagram (Correa and Lindstrom, 2012) obtained by replacing the standard deviation with the entropy and $\rho$ with MC (right panel). Mutual correlation was also calculated with respect to an underlying Gaussian distribution. The comparison clearly shows that entropy and, accordingly, MC are much more insensitive than $\sigma$ and $\rho$ to outliers applied to the original distribution of GPS IWV data. This supports the use of entropy and $\mathrm{MC}$ as tools to analyse a data set without the need to make assumptions on the underlying distribution function.

\subsection{Normalized entropy for integrated water vapour and vertical profiles}

Figure 4 compares the normalized entropies $H / \log n$, where $n$ is the number of states (histogram entries) retrieved for all instruments measuring IWV at the Lindenberg, Payerne, Potenza, and Southern Great Plains sites. For Lindenberg, Payerne, and Southern Great Plains at least four instruments are available; for Potenza, GPS IWV is available only from June 2011 and thus is not included in this study.

For the available measurements and in the range of atmospheric variability over the analysed stations there are no large differences in the uncertainties of IWV measurements. 

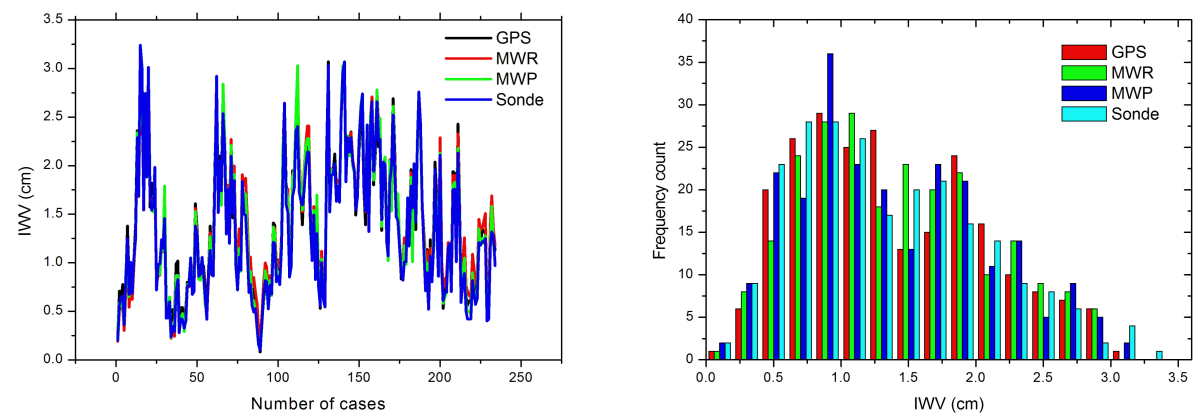

Figure 2. Example of the time series (left) of integrated water vapour obtained with the instruments available at the Lindenberg site (reported in Table 1) and histograms (right) of the time series shown in the left panel. After detrending of the time series, the histograms were used to calculate entropy and mutual correlation.
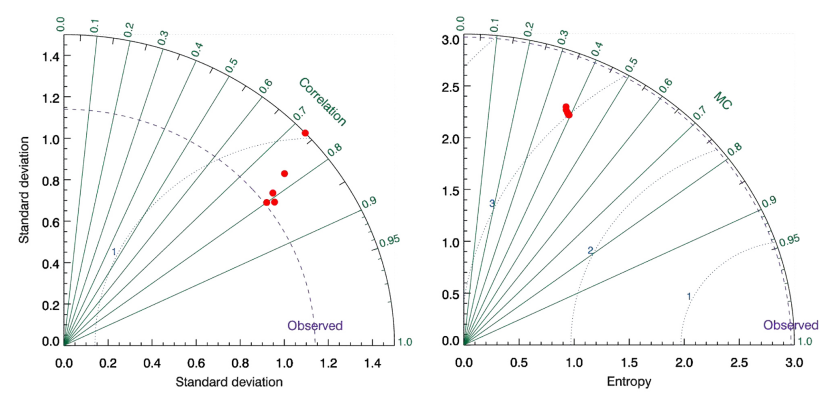

Figure 3. The left panel shows a Taylor diagram obtained for the GPS IWV time series collected at Lindenberg, and the same time series but adding to the IWV probability density function 5, 10, 20, 30 , and 40 outliers respectively. The correlation has been calculated with respect to an underlying Gaussian distribution fitted to 637 the data; in the right panel, a modified Taylor diagram is obtained by replacing the standard deviation with the entropy and $\rho$ with MC.

With the exception of Payerne, lidar entropy is the closest to radiosonde entropy, whether calibrated by using the sonde itself or the MWR. Moreover, at Payerne the lidar offers the lowest entropy of the instrument ensemble. At the SGP, GPS has the lowest entropy, though the values for all considered instruments are quite close. Similarly, at Lindenberg, where the MWR has the lowest entropy, all values are similar. At Potenza, the lowest entropy value is for the microwave profiler. As a whole, differences in the entropy of the time series between the different instruments are within $8 \%$. Obviously, the different atmospheric variability of each site can also result in large deviations between entropy values. This deviation could be smoothed if a longer temporal data set was investigated. Moreover, differences in the observation techniques and their experimental implementation (e.g. different measurement angles and fields of view) might also contribute to differences in the calculated entropies and to non-linear calibration drifts.

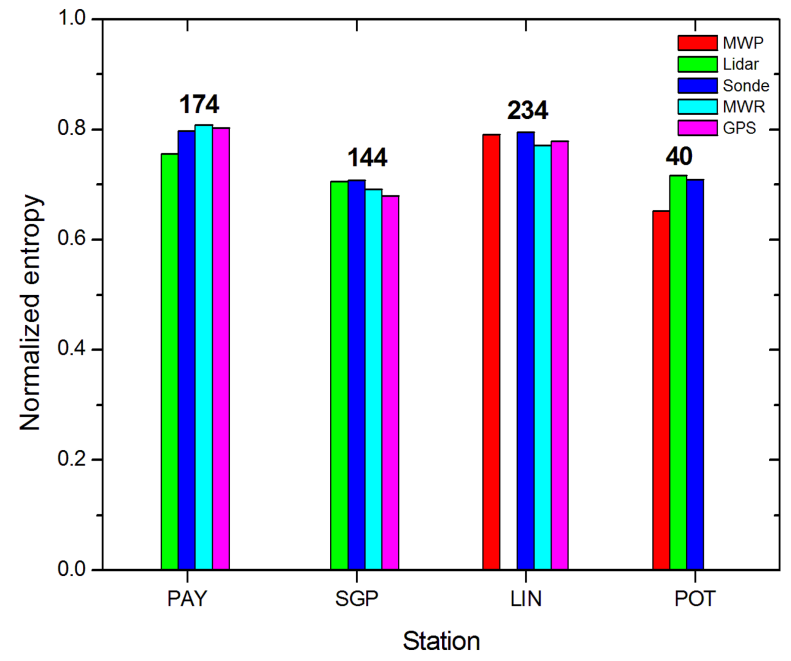

Figure 4. Comparison of the normalized entropy retrieved for the instruments measuring integrated water vapour at the Lindenberg (LIN), Payerne (PAY), Potenza (POT), and Southern Great Plains (SGP) sites. The data set considered includes all available measurements in 2010-2012. The numbers above the bars represent the number of cases selected, according to the quality assurance criteria for each station.

\subsection{Mutual correlation and distance for integrated water vapour and vertical profiles}

The statistical distance $D$, as defined in Eq. (5), is a dimensionless measure of the similarity of pairs of data points, data clusters or time series. Figure 5 compares the distances between the IWV time series from all instruments with the radiosonde series at the Lindenberg, Payerne, Potenza, and Southern Great Plains sites. The plot reveals very different results at different sites. In terms of the best performance of each instrument at the different sites, MWR has the highest redundancy and therefore the highest potential to reduce the uncertainty of the radiosonde IWV time series, with lidar, GPS and microwave profiler following. The distances 


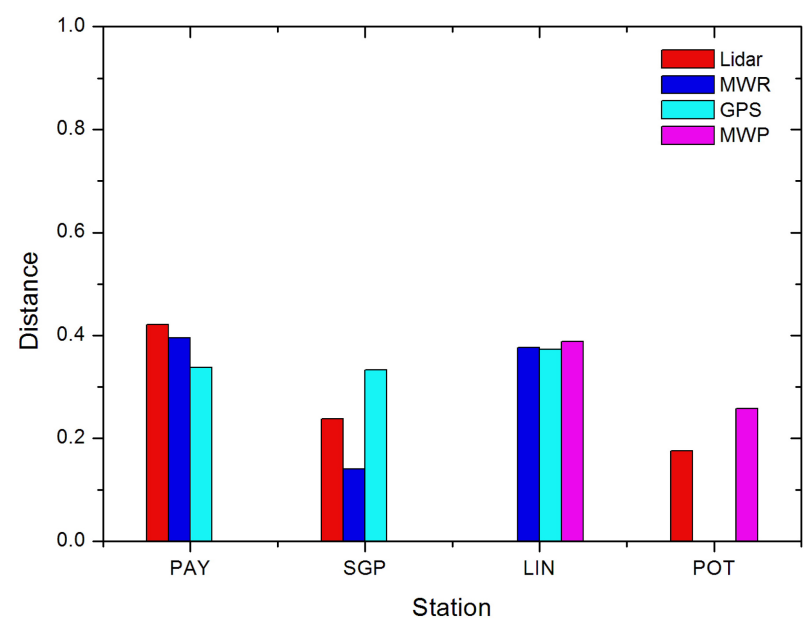

Figure 5. Comparison of the statistical distances between pairs of times series data retrieved for the instruments measuring integrated water vapour with respect to the time series obtained from the radiosondes at the Lindenberg (LIN), Payerne (PAY), Potenza (POT), and Southern Great Plains (SGP) sites. The data set considered includes all available measurements in 2010-2012.

from the radiosonde series are $>0.18$ for lidar, $>0.32$ for GPS, $>0.14$ for MWR, and $>0.28$ for microwave profiler. At Payerne and Potenza, all the techniques show good redundancy, though GPS IWV at Potenza is not included in the statistics, because the number of measurements is small for the considered period. However, criteria are needed to determine the acceptable levels of uncertainty and redundancy for a climate observation network. Section 3.5 deals with this aspect in more detail.

Normalized entropy and MC are compared for the available measurements of the water vapour or relative humidity $(\mathrm{RH})$ vertical profiles. In Fig. 6, the distance of the water vapour vertical profiles obtained with the Raman lidar (RL) and atmospheric emitted radiance interferometer (AERI) with respect to radiosonde (RS92) profiles are compared. Lidar profiles were retrieved by integrating signals over $10 \mathrm{~min}$ around the sonde launch time. The AERI profiles were averaged in the same time window. To improve the comparison among in situ, active and passive remote sensing measurements, the profiles from the three instruments were averaged over a vertical range of $1 \mathrm{~km}$. This should strongly reduce the differences related to instrument signal-to-noise ratio and to the effective vertical resolution, which differs for the different techniques. Moreover, for the AERI, the statistical retrieval provided by the ARM Archive (Turner and Loehnert, 2014) was considered; this retrieval is based on the radiosonde profile as a first guess, which affects the calculation of distance. Nevertheless, the comparison is provided to test the approach for passive profile retrievals. Figure 6 shows that, though the difference is small, AERI has lower values of distance along the entire profile, probably because of the use

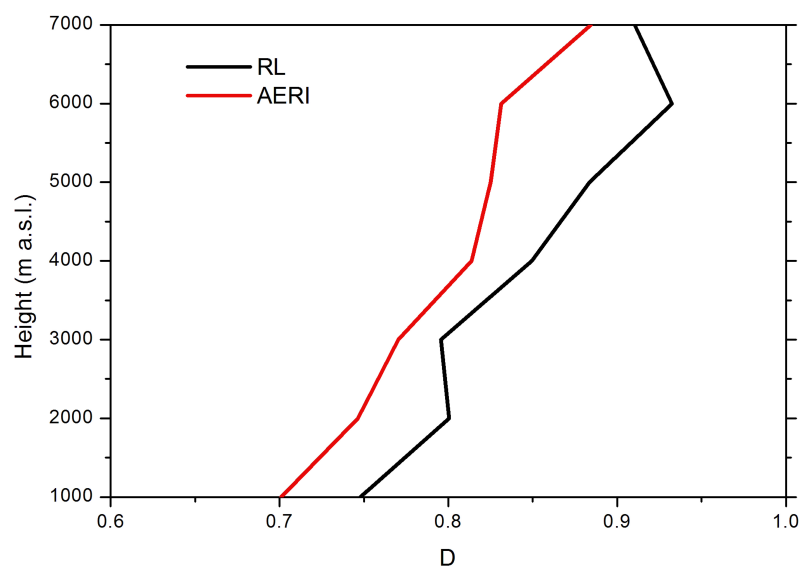

Figure 6. Comparison of the statistical distances of the Raman lidar (RL) and the atmospheric emitted radiance interferometer (AERI) time series from the RS92 radiosonde time series of water vapour vertical profile at the Southern Great Plains (SGP) site. The data set considered includes all measurements available at the SGP in 2010-2012, in 144 profiles.

of collocated radiosonde data as first guesses in the retrieval algorithm.

Figure 7 (left panel) compares the entropies computed for the RH profiles provided by RS92 radiosondes, Intermet radiosondes (I-Met), and CFH measuring in situ water vapour vertical profiles at SOD. Figure 7 (right panel) shows the profiles for one case on 15 March 2010. Because only 20 simultaneous profiles are included in this comparison, the calculated entropy values might underestimate the real uncertainty for the sensors. A larger data set should be considered for a full assessment of the differences in entropy for the various in situ measurements; this will be considered in future work, taking advantage of the data set available in the GRUAN network at the Boulder and Lindenberg sites. The comparison in the left panel of Fig. 7 reveals that the entropy values for all sensors differ by more than 0.2 from the ground to $2 \mathrm{kma}$.g.l., by less than about 0.1 from $2 \mathrm{~km}$ to $6 \mathrm{~km}$ a.g.l., and differ by more than 0.2 above. This observation indicates that RS92 and I-Met in situ measurements of atmospheric water vapour have a probabilistic uncertainty that differs from the CFH within $20 \%$ from most of the tropospheric range. $\mathrm{CFH}$ is considered the reference in situ profiling instrument (Suortti et al., 2008). The comparison of RH profiles in Fig. 7 (right) shows good agreement between RS92 and CFH, with a small bias affecting the RS92 in the free troposphere (Wang et al., 2013). On the other hand, the I-Met sondes are able to reproduce the vertical variability of the RH profile, but they are affected by a negative bias. Moreover, above $8 \mathrm{~km}$ the I-Met behaviour suddenly changes, overestimating RH. The comparison results are in agreement with literature values (WMO, 2010). The differences reported for the two sonde types are related to systematic effects on the $\mathrm{RH}$ profiles that contribute to 

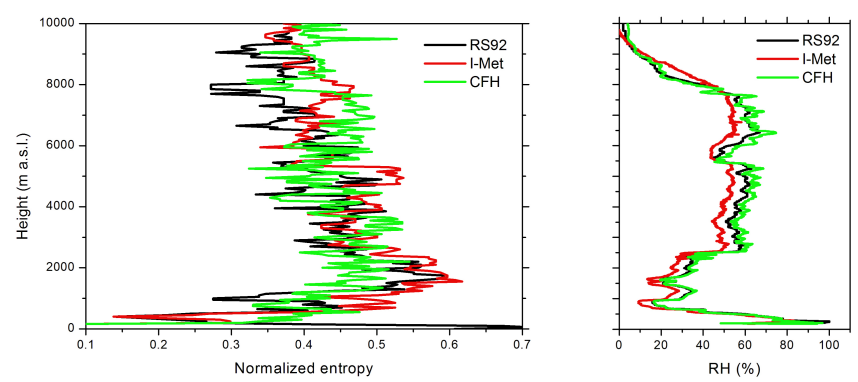

Figure 7. Comparison of the normalized entropy values (left) for the RS92 and Intermet radiosondes (I-Met) and the cryogenic frostpoint hygrometer $(\mathrm{CPH})$ measuring the in situ water vapour vertical profile at the Sodankyla site in 2010 (left panel); comparison of the relative humidity profiles in one case on 15 March 2010 (right panel).

the total uncertainty budget. This contribution can, in principle, be modelled and removed, but because of its systematic nature it cannot be evaluated with the entropy analysis discussed here. The presented analysis allows us only to state that the RH time series measured by the RS92, I-Met, and $\mathrm{CFH}$ show similar random uncertainty at all altitude levels below $10 \mathrm{~km}$.

\subsection{Conditional entropy}

The conditional entropy quantifies the amount of information needed to describe the outcome of a random variable $Y$, given the value of another random variable $X$. The conditioning usually reduces entropy. That is, given two time series $X$ and $Y$, the conditional entropy $H(X \mid Y) \leq H(X)$. Equality occurs only if $X$ and $Y$ are fully independent. Figure 8 shows the values of conditional entropy retrieved for most of the possible combinations of instruments measuring IWV at the SGP (upper panel) and POT (bottom panel) sites, for the data sets described above. In both plots, the values of the normalized entropies calculated for each single instrument are also shown as a comparison term to quantify the residual uncertainty affecting each instrument when one or more other instruments are assumed as good constraints. Figure 8 shows that the residual entropy obtained by conditioning one instrument with a second instrument is $30-40 \%$ lower than the entropy obtained for a single instrument. If two instruments are used for the conditioning, the residual entropy ranges between 5 and $20 \%$. This finding indicates that with reliable constraints, the entropy can be reduced by about $60-65 \%$ with respect to the use of a single instrument. The minimum residual uncertainties are obtained when the GPS is conditioned with the RL and the MWR at the SGP site, and when the $\mathrm{RL}$ is conditioned with the microwave profiler at the POT site.

These results also show that the residual uncertainties obtained with two conditioning constraints (two instruments) can be better than or similar to the value with only one instru-
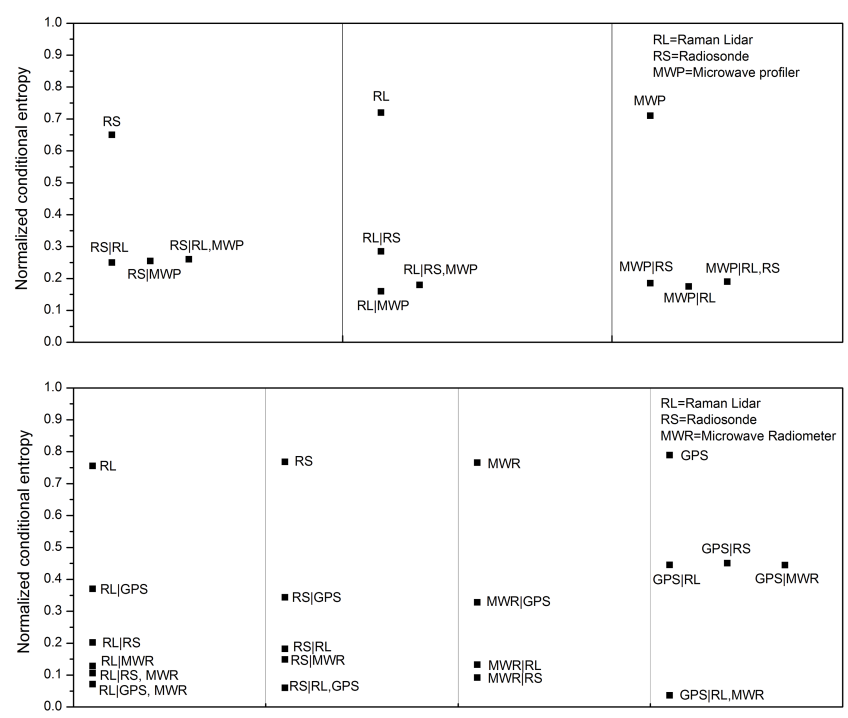

Figure 8. Comparison of the normalized conditional entropy values retrieved for most of the possible combinations of instruments measuring integrated water vapour at the Potenza site (upper panel) and the Southern Great Plains site (bottom panel).

ment as a constraint. This is relevant when synergetic products must be defined and retrieved by using algorithms that can integrate information from ground-based or satellite sensors. This is the case for all optimal estimation algorithms based on the Bayes' theorem, which is frequently adopted to improve atmospheric profiling. To quantify the effective advantages of integration, the presented analysis can be performed in advance of the elaboration of algorithms integrating measurements from different sensors. Moreover, conditional entropy can be applied similarly for directly measured quantities, like radiances, as well as for data products such as water vapour ground-based remote sensing. This is the case for algorithms making use of satellite measurements from polar and geostationary satellites to improve the resolution or reduce the uncertainty affecting the estimation of ECVs, but it is also true for algorithms merging satellite and groundbased passive sensor data to improve atmospheric profiling.

\subsection{Linear mutual correlation}

A comparison between MC and linear mutual correlation (LMC) provides a qualitative test for the non-linearity of the investigated problem. The plot in Fig. 9 shows a comparison between MC and LMC for the lidar and radiosonde at POT. In this case, both MC and LMC are normalized by the maximum entropy (the total number of entries in the joint histogram). The LMC underestimates the correlation between the two variables by about $10 \%$. Above $5.5 \mathrm{~km}$, the LMC overestimates the correlation by about $8 \%$, most likely because of the presence of outliers in the PDF. This example supports the use of MC as a more general concept than the 


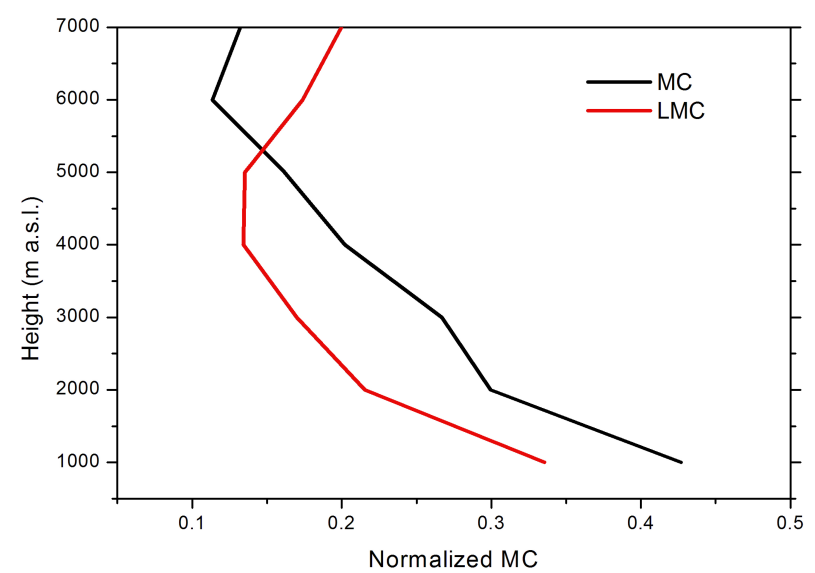

Figure 9. Comparison of the normalized mutual correlation for the linear (LMC) and non-linear cases (MC), calculated for the lidar and radiosonde data sets from the Potenza site.

LMC for quantifying the value of redundant measurements. The result is in agreement with outcomes from previous studies that analysed data sets including different types of data and compared Taylor's diagrams built by using standard deviation versus correlation and entropy versus MC (e.g. Correa and Lindstrom, 2012).

\subsection{Redundancy criteria}

The analysis above shows how to approach the problem of quantifying measurement redundancy by using the concepts of information theory. However, the usefulness of this approach can be clarified only if some criteria are identified to classify when two data sets are redundant. This obviously depends on the investigated variable and on the uncertainty limits assumed to be minimum requirements for studying a certain atmospheric process or climate trend.

Here, we present an example showing the relationship between distance values and the random uncertainty affecting IWV measurements. The aim is to clarify the use of MC and the related distance for quantifying redundant IWV measurements at GRUAN sites. The plot of Fig. 10 shows the distance between the radiosonde IWV time series at Lindenberg and the corresponding time series obtained by adding variable random noise to the radiosonde time series. The random noise is added to reproduce the effect of an additional random uncertainty, with relative values of $0-100 \%$ affecting an IWV time series with respect to the reference series. For example, a distance value lower than about 0.2 corresponds to a random uncertainty $20 \%$ larger than that of the original time series assumed as the reference. This example indicates a very simple way to approach data sets from different instruments or techniques, fixing a threshold consistent with the desired redundancy requirements. According to the GCOS requirements for the state-of-art capability, also reported in the GRUAN manual (http://www.wmo.int/pages/prog/gcos/

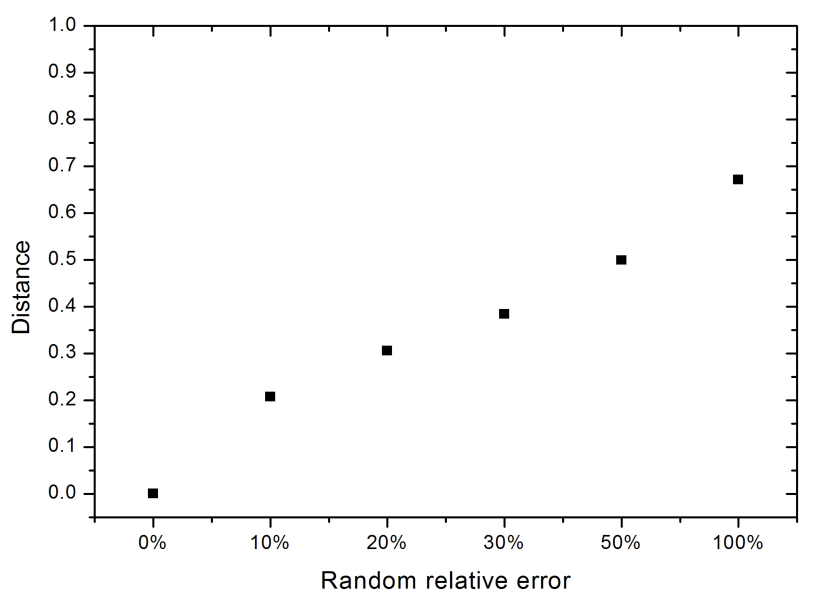

Figure 10. Statistical distance between the integrated water vapour time series retrieved from the radiosonde at the Lindenberg site and the corresponding time series obtained by adding random noise to the radiosonde time series to simulate the effect of increasing relative random uncertainty.

publications/gcos-171.pdf), atmospheric water vapour must be measured with a random error $<5 \%$ in the entire troposphere and stratosphere. This corresponds to a maximum random error $<5 \%$ affecting an IWV time series. If the random uncertainty is quantified by using entropy and the radiosonde IWV time series (the reference) is affected by random errors $<5 \%$, an IWV time series affected by a random error $<5 \%$ is consistent with the true series if the corresponding distance value is lower than about 0.2 (total random error $<10 \%$ ). The plot in Fig. 5 shows that the distance values for different instrument and different sites do not always meet this standard. The values $>0.2$ should be classified as not redundant in terms of the threshold of $5 \%$ random error affecting the two compared time series.

\section{Conclusions}

The ultimate aim of this study is to recommend the best combination of instruments for monitoring atmospheric water vapour. Though entropy and MC are robust concepts provided in information theory, representing appropriate metrics to quantify the uncertainty and redundancy of atmospheric measurements, they have never been applied extensively to climate data. In this paper, we show how entropy and MC can be used to evaluate the random probabilistic uncertainty in the ECV by analysing measurement redundancy.

The following conclusions can be drawn from the results of this study of data sets of water vapour from five GRUAN observation stations in 2010-2012:

1. The random uncertainty in the IWV time series obtained with the different instruments considered in this study 
(Raman lidar, GPS, MWR, microwave profiler, sondes) differs by $<8 \%$.

2. In terms of the best performances for each instrument at the different sites, the comparison of IWV time series showed that MWR have the highest redundancy and therefore the highest potential to reduce the random uncertainty of IWV time series as measured by radiosondes.

3. The distance between the time series of water vapour profiles at each altitude level has been also performed to show how to evaluate the redundancy of collocated in situ, active and passive profiling instruments, though for passive instruments this also depends on the retrieval algorithms and on which first-guess prior covariance is used.

4. Both RS92 and I-Met radiosondes can measure in situ atmospheric water vapour with the same random uncertainty as the $\mathrm{CFH}$, though the sondes are affected by a bias error that cannot be evaluated with the present approach.

5. A conditional entropy analysis showed that conditioning of the time series with more than one instrument, assumed as constraints, can decrease the residual entropy by at least $60 \%$ versus the use of one conditioning instrument. Moreover, the use of two conditioning instruments versus one results in similar or slightly better residual uncertainty.

6. An analysis of the relationship between distance and the random uncertainty showed that a maximum random error $<5 \%$ affecting the IWV estimated by two different techniques corresponds to a distance value less than about 0.2. That is, an IWV time series whose distance from a reference time series (i.e. IWV measured by radiosondes) is $>0.2$ exceeds the redundancy limits identified according to the GCOS criteria.

Final recommendations can be provided only if criteria to support a certain network are clearly defined according to the uncertainty thresholds assumed in the study of an ECV; however, the presented approach is versatile enough to be used with different data sets, stations, and instruments to provide the required feedback in terms of uncertainty and use of redundant measurements to reduce uncertainty in ECV values.

As a whole the concepts of entropy and mutual correlation demonstrate their potential if used as metrics for quantifying random uncertainty and redundancy in time series of atmospheric observations. The examples discussed in this work support the use of the mutual correlation as a more general concept than other linear metrics for the study of redundant measurements. Moreover, the analysis based on the entropy, $\mathrm{MC}$ and conditional entropy can be used for a preliminary feasibility study of the effective advantages obtained in using retrieval algorithms integrating measurements provided by different observation platforms, ground-based or satellite, both for direct measurements (e.g. radiances) and retrieved products (e.g. temperature, water vapour content, aerosol optical depth). For example, this is the case of those algorithms integrating measurements from different sensors using the Bayes' theorem (that is based on the concept of conditional probability) as well as for those algorithms integrating radiances measured by different sensor in different spectral ranges (e.g. Romano et al., 2007).

Acknowledgements. Data sources were as follows: ARM SGP data through the US Department of Energy (www.arm.gov); CIAO Potenza data through CNR-IMAA (http://www.ciao.imaa.cnr.it); Lindenberg data through the Lindenberg Meteorological Observatory, Richard Assmann Observatory, Deutscher Wetterdienst (http://www.dwd.de); PAY data through the Federal Office of Meteorology and Climatology MeteoSwiss (http://meteoswiss.admin.ch); SOD data through the Finnish Meteorological Institute (http://www.fmi.fi). The authors also gratefully acknowledge the useful comments of $\mathrm{A}$. Fassò from University of Bergamo. This work was supported by the US Department of Energy, Office of Science, Office of Biological and Environmental Research, under contract DE-AC02-06CH11357. The authors also acknowledge financial support for the ACTRIS Research Infrastructure Project supported by the European Union Seventh Framework Programme (FP7/2007-2013) under grant agreement no. 262254.

Edited by: K. Kreher

\section{References}

Adam, W., Dier, H., and Leiterer, U.: 100 years aerology in Lindenberg and first long-time observations in the free atmosphere, Meteorol. Z., 14, 597-607, 2005.

Arkhangel'skii, A. V. and Pontryagin, L. S.: General Topology I: Basic Concepts and Constructions Dimension Theory, Encyclopaedia of Mathematical Sciences, Springer, 1990,

Brocard, E., Philipona, R., Haefele, A., Romanens, G., Mueller, A., Ruffieux, D., Simeonov, V., and Calpini, B.: Raman Lidar for Meteorological Observations, RALMO - Part 2: Validation of water vapor measurements, Atmos. Meas. Tech., 6, 1347-1358, doi:10.5194/amt-6-1347-2013, 2013.

Calpini, B., Ruffieux, D., Bettems, J.-M., Hug, C., Huguenin, P., Isaak, H.-P., Kaufmann, P., Maier, O., and Steiner, P.: Groundbased remote sensing profiling and numerical weather prediction model to manage nuclear power plants meteorological surveillance in Switzerland, Atmos. Meas. Tech., 4, 1617-1625, doi:10.5194/amt-4-1617-2011, 2011.

Correa, C. and Lindstrom, P.: The Mutual Information Diagram for Uncertainty Visualization, International Journal for Uncertainty Quantification, 3, 187-201, 2012.

Cover, T. M. and Thomas, J. A.: Elements of Information Theory, John Wiley \& Sons, Inc., 1991. 
Dirksen, R. J., Sommer, M., Immler, F. J., Hurst, D. F., Kivi, R., and Vömel, H.: Reference quality upper-air measurements: GRUAN data processing for the Vaisala RS92 radiosonde, Atmos. Meas. Tech. Discuss., 7, 3727-3800, doi:10.5194/amtd-7-3727-2014, 2014.

Fassò, A., Ignaccolo, R., Madonna, F., Demoz, B. B., and FrancoVilloria, M.: Statistical modelling of collocation uncertainty in atmospheric thermodynamic profiles, Atmos. Meas. Tech., 7, 1803-1816, doi:10.5194/amt-7-1803-2014, 2014.

Hirsikko, A., O'Connor, E. J., Komppula, M., Korhonen, K., Pfüller, A., Giannakaki, E., Wood, C. R., Bauer-Pfundstein, M., Poikonen, A., Karppinen, T., Lonka, H., Kurri, M., Heinonen, J., Moisseev, D., Asmi, E., Aaltonen, V., Nordbo, A., Rodriguez, E., Lihavainen, H., Laaksonen, A., Lehtinen, K. E. J., Laurila, T., Petäjä, T., Kulmala, M., and Viisanen, Y.: Observing wind, aerosol particles, cloud and precipitation: Finland's new ground-based remote-sensing network, Atmos. Meas. Tech., 7, 1351-1375, doi:10.5194/amt-7-1351-2014, 2014.

Immler, F. and Sommer, M.: GRUAN-TD-4 Brief Description of the RS92 GRUAN Data Product (RS92.GDP), available at: http: //www.gruan.org, last access: 20 June 2014, 2010.

Immler, F. J., Dykema, J., Gardiner, T., Whiteman, D. N., Thorne, P. W., and Vömel, H.: Reference Quality Upper-Air Measurements: guidance for developing GRUAN data products, Atmos. Meas. Tech., 3, 1217-1231, doi:10.5194/amt-3-1217-2010, 2010.

Kitchen, M.: Representativeness errors for radiosonde observations, Q. J. Roy. Meteor. Soc., 115, 673-700, 1989.

Knuth, K. H.: Optimal data-based binning for histograms, arXiv preprint physics/0605197, 2013.

Madonna, F., Amodeo, A., Boselli, A., Cornacchia, C., Cuomo, V., D’Amico, G., Giunta, A., Mona, L., and Pappalardo, G.: CIAO: the CNR-IMAA advanced observatory for atmospheric research, Atmos. Meas. Tech., 4, 1191-1208, doi:10.5194/amt4-1191-2011, 2011.

Majda, A. and Gershgorin, J.: Quantifying uncertainty in climate change science through empirical information theory, P. Natl. Acad. Sci. USA, 107, 14958-14963, 2010.

Miller, M. A., Johnson, K. L., Troyan, D. T., Clothiaux, E. E., Mlawer, E. J., and Mace, G. G.: ARM value added cloud products: Description and status, in: Proc. of the 13th ARM Science Team Meeting, Broomfield, CO, ARM, available at: http://www.arm.gov/publications/proceedings/confl3/ extended_abs/miller-ma.pdf, last access: 20 June 2014, 2003.
Romano, F., Cimini, D., Rizzi, R., and Cuomo, V.: Multilayered cloud parameters retrievals from combined infrared and microwave satellite observations, J. Geophys. Res., 112, D08210, doi:10.1029/2006JD007745, 2007.

Seidel, D. J., Berger, F. H., Diamond, H. J., Dykema, J., Goodrich, D., Immler, F., Murray, W., Peterson, T., Sisterson, D., Sommer, M., Thorne, P., Vömel, H., and Wang, J.: Reference Upper-Air Observations for Climate: Rationale, Progress, and Plans, Bull. Am. Meteorol. Soc., 90, 361-369, doi:10.1175/2008BAMS2540.1, 2009

Suortti, T. M., Kivi, R., Kats, A., Yushkov, V., Kämpfer, N., Leiterer, U., Miloshevich, L. M., Neuber, R., Paukkunen, A., Ruppert, P., and Vömel, H.: Tropospheric Comparisons of Vaisala Radiosondes and Balloon-Borne Frost Point and Lyman- $\alpha$ Hygrometers during the LAUTLOS-WAVVAP Experiment, J. Atmos. Ocean. Tech., 25, 149-166, doi:10.1175/2007JTECHA887.1, 2008.

Thorne, P. W., Vömel, H., Bodeker, G., Sommer, M., Apituley, A., Berger, F., Bojinski, S., Braathen, G., Calpini, B., Demoz, B., Diamond, H. J., Dykema, J., Fassò, A., Fujiwara, M., Gardiner, T., Hurst, D., Leblanc, T., Madonna, F., Merlone, A., Mikalsen, A., Miller, C. D., Reale, T., Rannat, K., Richter, C., Seidel, D. J., Shiotani, M., Sisterson, D., Tan, D. G. H., Vose, R. S., Voyles, J., Wang, J., Whiteman, D. N., and Williams, S.: GCOS reference upper air network (GRUAN): Steps towards assuring future climate records, AIP Conf. Proc., 1552, 1042-1047, doi:10.1063/1.4821421, 2013.

Turner, D. D. and Loehnert, U.: Information content and uncertainties in thermodynamic profiles and liquid cloud properties retrieved from the ground-based Atmospheric Emitted Radiance Interferometer (AERI), J. Appl. Meteorol. Clim., 53, 752-771, doi:10.1175/JAMC-D-13-0126.1, 2014.

Wang, J., Zhang, L., Dai, A., Immler, F., Sommer, M., and Vömel, H.: Radiation Dry Bias Correction of Vaisala RS92 Humidity Data and Its Impacts on Historical Radiosonde Data, J. Atmos. Ocean. Tech., 30, 197-214, doi:10.1175/JTECH-D-12-00113.1, 2013.

WMO: TD - No. 1580: Instruments and Observing Methods Report No. 107, edited by: Nash, J., Oakley, T., Vömel, H., and Li, W., WMO Intercomparison of High Quality Radiosonde Systems, Yangjiang/China, 12 July-3 August 2010, 2010. 